\title{
Design a project of sewage plant to make the cross-section water quality reach standard
}

\author{
Lei Chao ${ }^{1}$, Bingtao Liu $^{2 *}$, Yinzhe Liu ${ }^{3}$ andYafeng $\mathrm{Li}^{4}$ \\ ${ }^{124}$ School of municipal and environmental engineering, Shenyang Jianzhu University, Shenyang, Liaoning Province, 110168, China \\ ${ }^{3}$ Tangshan Planning and Architectural Design \& Research Institute, Tangshan, Hebei Province, 063000, China
}

\begin{abstract}
According to regulations, the cross-section of Wangiiawopeng is regarded as a national control section of the Daling River. The water quality of the section must meet the surface water level III standard. According to the survey, the total phosphorus in the effluent from the sewage plant in Jianchang exceeds the standard is the main reason for the failure of the water quality. In order to make the cross-section water quality reaching standard, it is necessary to carry out the advanced treatment of phosphorus in the sewage plant. (1) The precipitation reaction of phosphorus and coagulant in sewage is strengthened by optimizing the type of coagulant, optimizing the dosage of coagulant, and selecting suitable coagulant aids. (2) The removal efficiency of phosphorus is improved by high-speed fiber filtration technology. (3) Phosphorus is removed by flocculation-sedimentation-high-speed fiber filtration technology. Experiments show that the SS in the sewage plant effluent is less than $5 \mathrm{mg} / \mathrm{L}$ and filter effluent TP is less than $0.2 \mathrm{mg} / \mathrm{L}$. The process construction investment is estimated to be 3.9 million yuan, and the operating electricity cost is estimated to be $0.2 \mathrm{cents} / \mathrm{m}^{3}$. The process has the characteristics of reliable processing technology, stable operation, easy maintenance.
\end{abstract}

\section{Introduction}

According to regulations, the cross-section of Wangjiawopeng is regarded as a national control section of the Daling River. The water quality of the section must meet the surface water level III standard. There is a sewage plant in Jianchang, which is five kilometers upstream of the section. The treated sewage is directly discharged into the Daling River, the river where the section is located and will flow through the section by natural degradation of the river. After the preliminary investigation, the total phosphorus in the effluent from the sewage plant is the main reason for the excessive phosphorus in the section. Therefore, only by improving the quality of the drainage, can the water quality of the section meet the standard.

\section{Materials and Methods}

\subsection{Chemical phosphorus removal technology}

At present, biological phosphorus removal and chemical phosphorus removal are mainly used in sewage plants ${ }^{[1]}$. Due to the limited efficiency of biological phosphorus removal and many influencing factors, to meet increasingly stringent sewage discharge standards, chemical phosphorus removal processes have gradually been widely adopted ${ }^{[2]}$.

Coagulation and flocculation are usually indispensable steps in sewage treatment. Therefore, the research on highefficiency coagulant and its coagulation mechanism is particularly important ${ }^{[3]}$.

Compared with inorganic low molecular coagulants, the flocs using inorganic polymer coagulants form faster and the particles are fuller, which makes the floc settling time shorter and easier to absorb colloidal particles in the sweeping water. Make the formed phosphate precipitate settle more completely. Therefore, the inorganic polymer coagulant has a better phosphorus removal effect.

\subsection{Process flow of deep phosphorus removal}

The process flow chart of deep phosphorus removal is shown in Figure 1. The effluent from the biochemical tank flows into the existing coagulation reaction tank, and sufficient (excessive) phosphorus removal agent is added to convert the residual dissolved total phosphorus in the water into phosphate precipitation; the phosphate precipitation will exist in the form of SS, and most SS will be removed through the existing sedimentation tank and sand filter, and then filtered by high-speed fiber filter device to further reduce the SS emission concentration to less than $5 \mathrm{mg} / \mathrm{L}$. The final effluent TP reached $0.2 \mathrm{mg} / \mathrm{L}$.

\footnotetext{
${ }^{1}$ e-mail: leichao@sjzu.edu.cn

${ }^{2 *}$ Corresponding author's e-mail: lansexueqiao@stu.sjzu.edu.cn
} 


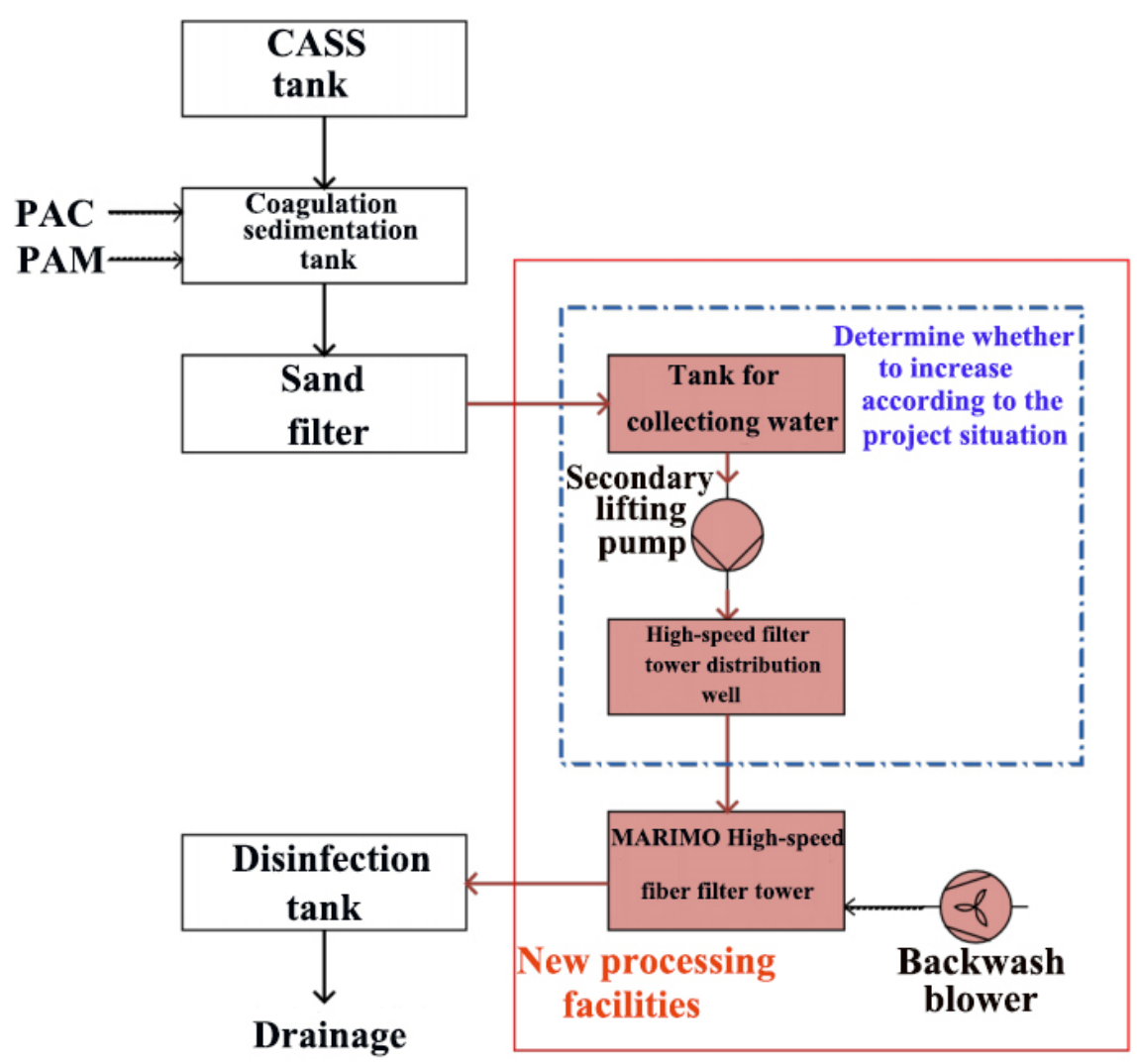

Figure 1. Process flow chart of deep phosphorus removal

\subsection{High-speed fiber filtration technology}

After coagulation and sedimentation, the tiny phosphorus flocs need to be intercepted by filtration. The interception effect of the filter unit on the flocs directly affects the phosphorus removal effect of the system.

High-speed fiber filtration technology is widely used in industrial wastewater, and it treats pollutants in the form of filtration, adsorption, and retention ${ }^{[4-6]}$. The high-speed fiber filter used in this project is a high-efficiency filter device made of heat-cured columnar fiber particle materials. The high-speed fiber filter tower adopts thermosetting molding granular fiber filter material, which is made of polyester resin. There is no need to replace the filter material regularly. The actual project has verified that the service life of the fiber filter material can reach 20 years, and the annual replenishment is less than $1 \%$. This device adopts gravity-type deep-layer filtration with a designed filtration speed of $1000-1500 \mathrm{~m} / \mathrm{d}$ and high filtration efficiency. The system has the characteristics of low head loss, low backwash water volume, small footprint, high SS removal rate, a high degree of automation in operation, and low operating and maintenance costs. The comparison between fiber filtration technology and ordinary sand filter technology is shown in Table 1.

Table 1. Comparison between fiber filtration technology and common sand filtration technology

\begin{tabular}{ccc}
\hline & High-speed fiber Filtration technology & Sand filter technology \\
Filtration rate & $1000 \mathrm{~m} / \mathrm{d}$ & $200 \sim 300 \mathrm{~m} / \mathrm{d}$ \\
SS Catch & $6 \mathrm{~kg}-\mathrm{SS} / \mathrm{m}^{3}$ & $4 \mathrm{~kg}-\mathrm{SS} / \mathrm{m}^{3}$ \\
SS Content in filtered water & $\leq 5 \mathrm{mg} / \mathrm{L}$ & $\leq 8 \mathrm{mg} / \mathrm{L}$ \\
SS Removal rate & $50 \sim 80 \%$ & $40 \sim 70 \%$ \\
Installation area & $1 / 2 \sim 1 / 3$ of sand filter technology \\
Operation and maintenance cost & Equivalent to sand filtration technology & - \\
\hline
\end{tabular}




\section{Results \&Discussion}

\subsection{Phosphorus composition in the effluent of the sewage plant}

Analyzing the phosphorus composition in the effluent of the sewage plant, it was found that the concentration of

Table 2. Test results of effluent coagu

\begin{tabular}{ccccc}
\hline Project & Unit & 1\#experiment & 2\#experiment & 3\# experiment \\
\hline Raw water ${ }^{[1]}$ capacity & $\mathrm{ml}$ & 500 & 500 & 500 \\
Raw water TP & $\mathrm{mg} / \mathrm{L}$ & 0.35 & 0.35 & 0.35 \\
Solubility of raw water TP & $\mathrm{mg} / \mathrm{L}$ & 0.25 & 0.25 & 0.25 \\
PAFC Add amount & $\mathrm{mg} / \mathrm{L}$ & 8.95 & 9.94 & 11.93 \\
Al/P & & 4.5 & 5 & 6 \\
PAM & $\mathrm{mg} / \mathrm{L}$ & 0.5 & 0.5 & 0.5 \\
Supernatant ${ }^{[2]} \mathrm{TP}$ & $\mathrm{mg} / \mathrm{L}$ & 0.09 & $\leq 0.08$ & $\leq 0.08$ \\
Supernatant inorganic & $\mathrm{mg} / \mathrm{L}$ & $\leq 0.05$ & Not detected & Not detected \\
phosphorus ${ }^{[3]}$ & & $73 \%$ & $77 \%$ & $77 \%$ \\
TP Removal rate & & &
\end{tabular}

[1] Raw water is the effluent of the wastewater treatment plant

[2] The supernatant was taken from the coagulation sedimentation for $30 \mathrm{~min}$

[3] Inorganic phosphorus is directly measured without digestion

According to the analysis of the experimental results:

(1) Adding PAFC and PAM can convert most of the soluble TP into phosphate precipitation, indicating that the current content of organic phosphorus in sewage plant effluent is relatively low, and the conversion of organic phosphorus to inorganic phosphorus is relatively complete in the secondary biochemical treatment process.

(2) The amount of PAFC used in the existing coagulation sedimentation system of the sewage plant is insufficient, or the content of the active ingredient of PAFC is low.

(3) After the coagulation reaction, the TP removal rate is greater than $70 \%$. When the effluent TP is $0.35 \mathrm{mg} / \mathrm{L}$, the solubility TP after the coagulation reaction is about $0.1 \mathrm{mg} / \mathrm{L}$, and another TP is in the form of phosphate precipitation(SS). Therefore, to meet the $0.2 \mathrm{mg} / \mathrm{L}$ TP emission requirement, the SS removal rate needs to be increased.

Therefore, this plan suggests adopting technical methods such as optimizing the type of coagulant, optimizing the dosage of coagulant, and selecting suitable coagulant aids in the coagulation process unit of the organic phosphorus in the effluent was about $0.06 \mathrm{mg} / \mathrm{L}$, accounting for about $18 \%$ of TP.

Coagulation beaker experiment on sewage plant effluent. Compound coagulant PAFC and PAM. Among them, PAFC: drinking grade, $\mathrm{Al}_{2} \mathrm{O}_{3}$ content 29\%, configuration mass concentration $10 \%$; PAM: configuration mass concentration $0.1 \%$, dosage is $0.5 \mathrm{mg} / \mathrm{L}$. The coagulation beaker test is shown in Table 2 . existing sewage plant to strengthen the phosphorus and coagulant precipitation reaction in the sewage. The coagulant, coagulant aids, coagulation conditions, $\mathrm{pH}$, and stirring speed used in the project need to be tested to determine the optimal flocculation and sedimentation conditions. In practical applications, the combination of chemical factors (solubility product, $\mathrm{pH}$ ) and physical factors (temperature, stirring, fluid mechanics) also have a significant impact on the flocculation effect.

\subsection{Effect of micro-flocculation and high-speed fiber filtration on phosphorus removal}

After PAFC is used as a coagulant in the water, a part of the PAFC combines with the soluble phosphate in the water to form a precipitate. At the same time, PAFC rapidly hydrolyzes to form a complex, which adsorbs suspended particles and colloids in the water, and is finally intercepted from the water by the subsequent fiber filtration process. It can be seen from Figure 2 that when the PAFC dosage is zero, the removal rate of TP by the original process is $41.18 \%$. As the PAFC dosing concentration increases, the removal rate of $\mathrm{TP}$ also increases. When the PAFC concentration was $12 \mathrm{mg} / \mathrm{L}$, the TP removal rate reached a maximum of $88.64 \%$. At this time, the TP concentration of the effluent is below $0.1 \mathrm{mg} / \mathrm{L}$, which meets the target water quality. 


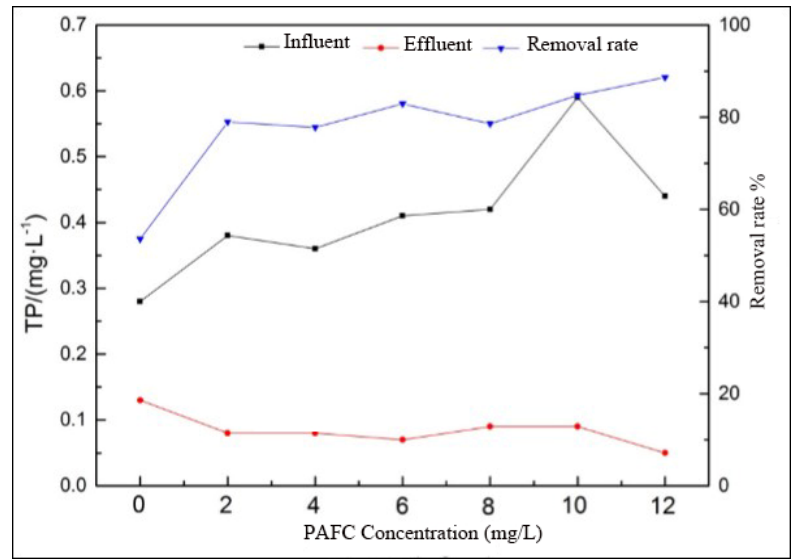

Figure 2. TP removal efficiency under different PAFC concentration

As shown in Figure 3, the combination of PAFC and PAM has a relatively stable effect on TP removal. After adding different concentrations of PAM, the removal of TP did not change much and remained $83 \% \sim 85 \%$. Adding different concentrations of PAM did not significantly improve the removal effect of TP. This is because the concentration of TP and SS in the water is too low, the ability of coagulation to remove phosphorus is close to the limit. Moreover, as an organic polymer coagulant, if PAM is added to water in excess, the water will become viscous, which is not conducive to the subsequent high-speed fiber filtration device.

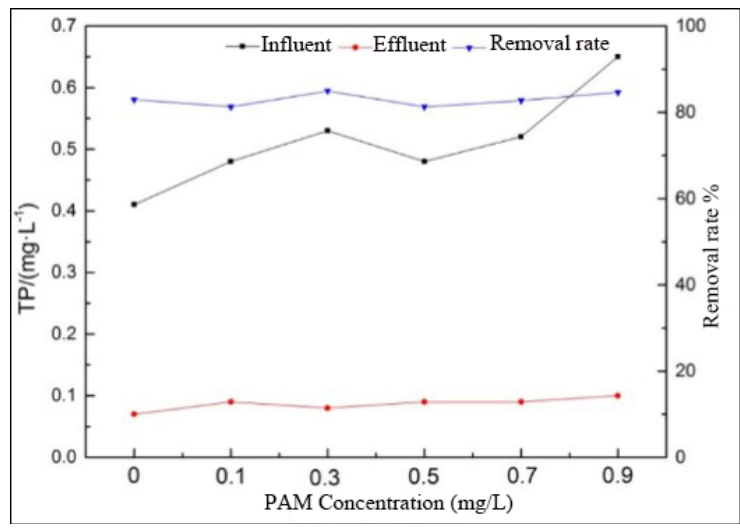

Figure 3. TP removal efficiency of $6 \mathrm{mg} / \mathrm{L}$ PAFC and PAM with different concentrations

\subsection{Pilot study on flocculation-sedimentation- high-speed fiber filtration for phosphorus removal}

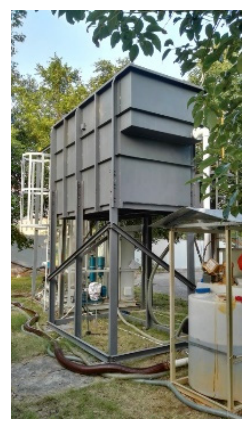

In response to the demand for phosphorus removal in the advanced treatment of sewage treatment plants, a pilot study was carried out. The research device is shown in Figure 4.

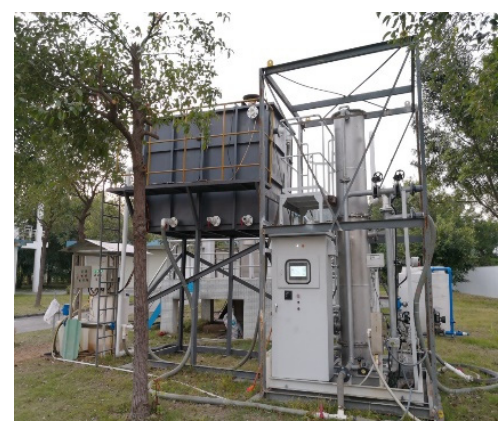

Figure 4. Pilot plant

The results of the pilot study showed that under the condition of a sufficient dosage of phosphorus removal agent, the total dissolved phosphorus was basically transformed into phosphate precipitation. Figure 5 shows the relationship between total phosphorus in filtered water and SS. When the effluent SS of the high-speed fiber filter device is less than $5 \mathrm{mg} / \mathrm{L}$, the filtered effluent TP can reach less than $0.2 \mathrm{mg} / \mathrm{L}$. 


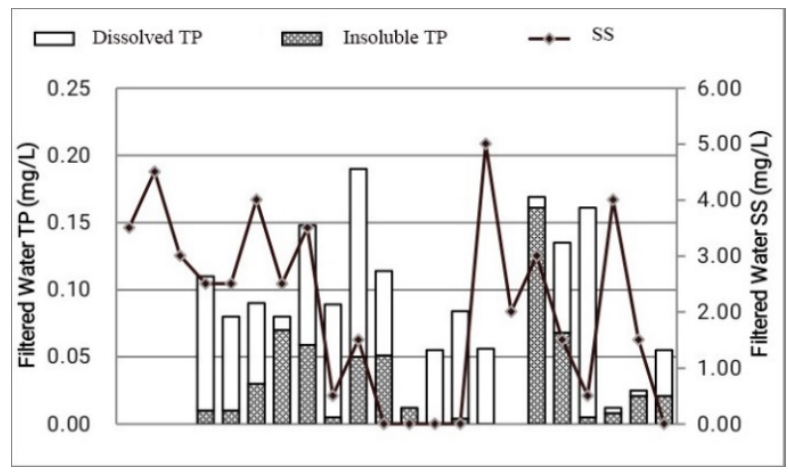

Figure 5. Relationship between total phosphorus and SS in filtered water

\subsection{Construction investment estimate}

The first phase of the project uses 4 integrated high-speed fiber filter towers, without civil engineering. After the equipment is transported to the site, it can be commissioned and operated after the water and electricity connections are completed. The sewage plant with a treatment scale of $30,000 \mathrm{t} / \mathrm{d}$ is to build a fiber filter device, and the required filter material is $11.4 \mathrm{~m}^{3} / 10,000$ tons of water. The price of the new fiber filter material is 30,000 yuan $/ \mathrm{m}^{3}$, and the new fiber filter material requires a total of 1.026 million yuan. Fiber filter tower, auxiliary, and control equipment, and technical services are 2.9 million yuan. The total investment is 3.926 million yuan.

\subsection{Running electricity cost estimate}

In the first stage of the deep phosphorus project of the sewage plant, the high-speed fiber filter device adopts gravity water intake and air-water combined reverse cleaning. Use raw water as cleaning water, no need to set up a water inlet pump, and reverse cleaning water pump. The main electrical equipment is a backwash blower. The system has a high degree of automation, good operation stability, and low failure probability, which greatly reduces the workload of equipment maintenance and repair. The main operating cost of the high-speed fiber filtration system comes from power consumption. The cost analysis of the operating power consumption of the high-speed fiber filtration system of this project is as follows.

The power of the backwash blower is $15 \mathrm{~kW}$. Each filter tank should be backwash 1-2 times a day and considered as 2 times. Each backwashing requires 15-20 minutes of air blast, which is considered as $30 \mathrm{~min} / \mathrm{time} .4$ filter tanks, blower running hours per day: $0.5 \mathrm{~h} \times 2 \times 4=4 \mathrm{~h}$. Daily power consumption: $15 \mathrm{~kW} \times 4 \mathrm{~h}=60 \mathrm{kWh}$. The electricity price is considered at 0.8 yuan $/ \mathrm{kWh}$, and the treated water volume is calculated at $30000 \mathrm{~m}^{3} / \mathrm{d}$. The energy consumption of one cubic meter of water is $0.002 \mathrm{kWh}$. The electricity fee is 0.0016 yuan $/ \mathrm{m}^{3}$. Besides, consider the power consumption of electric valves and automatic control cabinets. The electricity fee is 0.00224 yuan $/ \mathrm{m}^{3}$.

\section{Conclusion}

According to the specific conditions of the cross-section of Wangiiawopeng in Daling River, the water environment of the section was analyzed, and the contribution of the sewage plant's drainage to the pollution of the section was clarified. If completed by the water quality improvement plan, it is the main pollutant TP can be reduced to meet the surface water Class III water standard, and finally, meet the requirements of cross-section compliance. The following conclusions are drawn:

(1) Excessive total phosphorus in wastewater from the sewage plant is the main reason that affects the crosssection standard.

(2) Coagulation with high-speed fiber filtration to remove phosphorus. Using PAFC as a coagulant and increasing the dosage of PAFC can strengthen the precipitation reaction of phosphorus and coagulant in sewage, which is beneficial to the removal of TP. Under the condition of a sufficient dosage of phosphorus removal agent, the effluent of the high-speed fiber filter device can reach $\mathrm{SS}<5 \mathrm{mg} / \mathrm{L}$, and the filtered effluent can reach $\mathrm{TP}<0.2 \mathrm{mg} / \mathrm{L}$.

\section{Acknowledgments}

This research was funded by the China National Critical Project for Science and Technology on Water Pollution Prevention and Control (2018ZX07601-002), to which the authors express their thanks.

\section{References}

1. Fang. J.F. (2017) Experimental study on chemically enhanced biological phosphorus removal in municipal sewage treatment plants. Chemical Management,33:122-125

2. BELLIER N, CHAZRENC F, COMEAU Y. (2006) Phosphorus removal from wastewater by mineral apatite. Water Research, 40(15): 2965-2971.

3. Yu R, Sun L, Sun T. (2012) Study on the flocculating mechanism of microbial flocculants overview and illustration. Journal of safety and Environment, 12(1):24-26.

4. Liao. Y.H., Cheng. Q.P., Deng.F. (2016) Semi-coke 
adsorption of heavy metals in aquaculture wastewater. Journal of Environmental Engineering, 10(4):18421846.

5. Zhou. S.Y., Yang.K., Wu. X.F. (2016) Research progress on the mechanism of constructed wetland plants to remove heavy metals in wastewater. Wetland Science, 14 (5):717-724.

6. Li. H.X, Qin. Z.H, Lu.Y.(2018) Study on the influence of $\mathrm{pH}, \mathrm{H} 2$, and $\mathrm{H} 2 \mathrm{~S}$ on the degradation of organic matter in anaerobic digestion. Resource conservation and environmental protection, 4:82-83. 